\title{
Application of multi-stage Monte Carlo method for solving machining optimization problems
}

\author{
Miloš Madić $^{a^{*}}$, Marko Kovačević ${ }^{b}$ and Miroslav Radovanović ${ }^{a}$
}

${ }^{a}$ Faculty of Mechanical Engineering, University of Niš, A. Medvedeva 14, Niš, Serbia

${ }^{b}$ Faculty of Electronic Engineering, University of Niš, A. Medvedeva 14, Niš, Serbia

\section{H R O N I C L E}

Article history:

Received October 262013

Received in Revised Format

July 52014

Accepted July 52014

Available online

July 82014

Keywords:

Monte Carlo method

Multi-stage

Machining

Optimization

Meta-heuristics \begin{abstract}
A B S T R A C T
Enhancing the overall machining performance implies optimization of machining processes, i.e. determination of optimal machining parameters combination. Optimization of machining processes is an active field of research where different optimization methods are being used to determine an optimal combination of different machining parameters. In this paper, multi-stage Monte Carlo (MC) method was employed to determine optimal combinations of machining parameters for six machining processes, i.e. drilling, turning, turn-milling, abrasive waterjet machining, electrochemical discharge machining and electrochemical micromachining. Optimization solutions obtained by using multi-stage MC method were compared with the optimization solutions of past researchers obtained by using meta-heuristic optimization methods, e.g. genetic algorithm, simulated annealing algorithm, artificial bee colony algorithm and teaching learning based optimization algorithm. The obtained results prove the applicability and suitability of the multi-stage MC method for solving machining optimization problems with up to four independent variables. Specific features, merits and drawbacks of the MC method were also discussed.
\end{abstract}

\section{Introduction}

In recent years, high resource efficiency and optimization of machining processes have been vital for machining companies in order to gain a competitive advantage on the market. Machining processes are highly complex processes affected by a number of input variables, i.e. machining parameters, which in consort have an important role on different process performance characteristics such as quality, cost, productivity, processing time, etc. In real production environment, the selection of machining parameter settings is usually made based on experience and knowledge of a machinist (or a production planner), machining handbooks and manufacturers' recommendations (Rao \& Pawar, 2010; Rao \& Kalyankar, 2013). Although this is essentially a subjective approach, and chosen values of machining parameters are preferably conservative, or even not near optimal ones, in some cases this approach is sufficient. However, many process parameters and the complex and stochastic nature of the machining processes mean that achieving the optimal performance, even for a highly skilled machinist with a state-of-the-art machine tool, is rarely possible (Rao \& Pawar, 2009). The most adverse effect of such approach may lead to product quality deterioration, increase in operation cost and machining time, 
decrease in productivity, etc. In order to gain a competitive advantage on the fierce market, get the most from a machine tool and enhance the overall machining efficiency, it is necessary to select machining parameter settings more intelligently. Recent studies indicate that the selection of machining parameter settings is often performed in a two-stage approach consisting of mathematical modeling of a machining performance characteristic and optimization using an optimization method. Mathematical modeling is aimed at establishing mathematical relationships between machining parameters and different performance characteristics. Since many machining optimization problems are complex, highly non-linear and multi-dimensional, meta-heuristic optimization methods have become a preferred trend for solving machining optimization problems.

Meta-heuristic optimization methods conceptually developed to imitate natural phenomena, the biological evolutionary process (genetic algorithm-GA), the physical annealing process (simulated annealing-SA), social behavior of bird flocking (particle swarm optimization-PSO), behavior of real ant colonies (ant colony optimization-ACO), artificial bee colony (artificial bee colony-ABC), have been employed instead of classical optimization methods such as sequential unconstrained minimization technique, feasible direction method, goal programming and non-linear programming. Actually, as noted by Yildiz (2009), the complexity of machining problems creates the requirement for increasingly effective optimization algorithms. Many researchers and authors have found classical optimization methods ineffective for solving machining optimization problems because of the following: a tendency to obtain a local optimal solution (Rao \& Pawar, 2010), very complex nature and inability to handle multi-objective problems effectively (Rao \& Kalyankar, 2013) and lack of robustness (Rao \& Pawar, 2009; Rao \& Pawar 2010). As noted by Mukherjee and Ray (2006), researchers and practitioners prefer an alternative cost effective near-optimal (or approximate) solution than the exact optimal one, as it may be extremely difficult to find the exact optimal point in the high dimensional and multimodal search space. Rao et al. (2008) used the PSO algorithm to determine optimal machining parameter settings for an electro-chemical machining (ECM) process and compared its performance with that obtained by other optimization methods. Rao and Pawar (2009) applied the ABC algorithm in order to determine optimal machining parameter values for a wire electric discharge machining (WEDM) process. Rao and Pawar (2010) developed a comparative study of the application of the ABC, SA and PSO algorithm in order to obtain the optimum machining parameter values for various cutting strategies in multi-pass milling process. In the research of Samanta and Chakraborty (2011), the ABC algorithm was applied to search out the optimal machining parameter values combinations of three non-conventional machining processes. The obtained single and multi objective optimization solutions were better compared to optimization solutions obtained by the past researches.

GA was considered by Kilickap et al. (2011) to optimize drilling of AISI 1045 steel. In the study, three machining parameters such as cutting speed, feed rate, and cutting environment were employed. Response surface methodology was used to develop mathematical model and GA was used to determine optimal machining conditions for ensuring the minimum surface roughness. Bhushan et al. (2012) presented the GA approach for optimization of surface roughness in machining of Al alloy SiC particle composite material. A surface roughness prediction model was developed in terms of four machining parameters such as cutting speed, feed rate, depth of cut and tool nose radius. The authors concluded that the GA method outperformed the RSM regarding the best (minimal) surface roughness predicted value. Savas and Ozay (2008) applied GA for optimization of the surface roughness in the process of tangential turn-milling process. The mathematical model consisted of four independent variables, i.e. machining parameters, such as depth of cut, workpiece speed, tool speed and feed rate. Zain et al. (2011) applied GA and the SA algorithm to optimize the machining parameter values of abrasive waterjet (AWJ) machining process. Five machining parameters were considered in the research including traverse speed, waterjet pressure, standoff distance, abrasive grit size and abrasive flow rate. Comparative analysis of optimization solutions indicated that SA provided better solutions than GA. However, recently Yusup et al. (2012) attempted the same optimization problem and observed that the ABC algorithm outperformed both SA and GA. Rao and Kalyankar (2013) applied a 
newly developed advanced algorithm named 'teaching-learning-based optimization (TLBO) algorithm' for optimization of non-conventional machining processes such as ultrasonic machining (USM), abrasive jet machining (AJM) and WEDM. It was concluded that TLBO has given a considerable improvement in terms of results and convergence over GA, SA, ABC and PSO. Recently, Madić and Radovanović (2014b) proposed the use of pattern search (PS) algorithm, as deterministic direct search method, for solving single- and multi-objective machining optimization problems. While solving several case studies it has been found that the PS algorithm is an efficient optimization method showing good competitive potential against stochastic direct search methods such as meta-heuristic algorithms.

From the literature review, it has been observed that GA, SA, PSO, ABC and ACO have been extensively used for solving machining optimization problems (Yusup et al., 2012). It is observed that meta-heuristic algorithms were identified as effective stochastic optimization methods for solving machining optimization problems. However, the well-known difficulty in determination of optimum algorithm specific controlling parameters such as crossover and mutation rate in the case of GA, initial temperature in the case of SA, inertia weight in the case of PSO, etc., affects the effectiveness of metaheuristic optimization methods in searching for global optimal solutions (Rao \& Kalyankar, 2013). As noted by Bhushan et al. (2012), there is no guideline yet given by the researchers which could be followed in recommending the best combination of parameter values for the best optimal solution. In addition, application of meta-heuristic algorithms implies that the repeatability of optimization results obtained with the same initial condition settings is not guaranteed (Madić et al., 2013). Meta-heuristic optimization methods perform efficient and comprehensive exploration of the optimization search space using the random Monte Carlo (MC) search guided by governing mechanisms which imitate certain strategies taken from nature. GA, SA and recently the $\mathrm{ABC}$ algorithm have shown their strength in this respect. Recently, it has been shown that the iterative exhaustive search algorithm has the potential for solving the aforementioned machining optimization problems for a given discrete optimization space (Kovačević et al., 2013). However, to the authors' knowledge there is no application of the MC method for solving machining optimization problems, which is traditionally applied to continuous optimization problems. As noted by Mosegaard and Sambridge (2002), MC methods are powerful optimization tools when searching for globally optimal solutions amongst numerous local optima.

The main motivation behind this paper is to investigate the applicability and efficiency of a conceptually simple MC method for solving machining optimization problems. Although the aforementioned meta-heuristic optimization methods have proved that they can succeed in obtaining good optimization solutions, the average process engineer in real machining environment may not feel familiar with these methods, which require deeper knowledge of artificial intelligence and optimization. Moreover, in some cases, specialized software or programming skills are needed. With an increasing number of meta-heuristic optimization methods and their combinations that have emerged in recent years, it has become very difficult even for researchers to become familiar with all these optimization methods. Because of these reasons, as noted by Besseris (2008), in practice only the simplest of optimization tools are eventually proved workable in the factory. In that sense, this paper is an attempt to investigate the applicability of the MC method for solving machining optimization problems. The proposed optimization procedure was employed to search out the optimal combinations of machining parameters for six machining processes, i.e. drilling, turning, turn-milling, AWJ machining, electrochemical discharge machining (ECDM), and electrochemical micromachining (EMM). The obtained optimization solutions were compared with the optimization solutions obtained by the past researchers using meta-heuristic algorithms.

\section{Machining optimization}

Optimization of machining processes has been of considerable interest in the literature. The ultimate goal of machining optimization is to select machining parameter values such that the overall machining 
performance is enhanced. Depending on the criteria, optimization of machining processes should result in an increase in product quality, increase in productivity, decrease in machining cost and time, etc. Mathematically, optimization of machining processes is related to optimization of an objective function, often representing a machining performance, under some machining parameter constraints. The machining optimization problem may be expressed as follows:

$$
\begin{aligned}
& \text { Minimize(or maximize) } f(x) \\
& \text { subject to : } g_{i}(X) \leq 0, i=1 \ldots m \\
& \qquad X_{j}^{l} \leq X_{j} \leq X_{j}^{u}, j=1 \ldots n
\end{aligned}
$$

where $\boldsymbol{X}$ is the vector of machining parameters, $f(x)$ is the objective function to be minimized (maximized), $g_{i}(X)$ is the $i$-th functional constraint, and $X_{j}^{l}$ and $X_{j}^{u}$ are the lower and upper bounds of $j$-th machining parameter $X_{j}$.

Generally, objective functions for machining optimization problems can be developed in two ways either analytically or empirically. The development of analytical equations, based on basic laws and principles of a machining process, requires deeper knowledge and understanding of the machining process and is usually quite complex. These equations are more general, but less accurate since they involve some simplifications and approximations in relation to the real machining process and do not take into account any imperfections in the process. Therefore, analytical solutions for describing machining processes are generally not accurate enough for practical usage (Davim, 2001).

Compared with analytical approach, empirical model development does not need deeper knowledge of the phenomena occurring in the machining process, but the development of complex mathematical models and designs implies a more profound knowledge of theory of experimental design and mathematical modeling. The development of empirical models comprises the selection of experimental design, realization of experiment, data collection and development of mathematical equations using different modeling techniques, e.g. regression analysis, artificial neural networks etc. Theory and practice in the field of machining have shown that in most cases the best choice is a mathematical model in the form of polynomials (linear, quasi-linear, square, etc.) (Savas \& Ozay, 2008).

\section{Monte Carlo Method}

The term Monte Carlo method (MC method) was coined in the 1940s by physicists S. Ulam, E. Fermi, J. von Neumann and N. Metropolis under the name "statistical sampling". Since the pioneer studies in the 1940s and 1950s, this method has been applied in almost all areas of science (Yang, 2010). MC methods have been used for a long time, but only in the last few decades, the methods have gained the status of fully rounded numerical methods. As for obtaining a reasonably accurate assessment, the calculation of large number of special cases is needed, as well as appropriate statistical analysis, the efficient application of the MC methods begins with the emergence of high-speed computers able to perform fast computations (Madić \& Radovanović, 2014a).

At the heart of any Monte Carlo method there is a random number generator: a procedure that produces

an infinite stream: $U_{1}, U_{2}, U_{3}, \ldots \sim$ iid Dist of random variables that are independent and identically distributed (iid) according to some probability distribution Dist. When this distribution is the uniform distribution, that is Dist $=U(0,1)$, the generator is said to be uniform random number generator (Kroese et al., 2011). Monte Carlo methods can be divided into two categories: the sampling methods and the optimization methods. Monte Carlo sampling is useful when the space of feasible solutions is to be explored, and measures of resolution and uncertainty of solution are needed. Monte Carlo optimization methods are powerful tools when searching for globally optimal solutions amongst numerous local optima (Mosegaard \& Sambridge 2002). The optimization based on Monte Carlo 
methods can be useful for solving optimization problems with many local optima and complicated constraints, possibly involving a mix of continuous and discrete variables (Kroese et al., 2011). When using the MC method for solving optimization problems there are basically two approaches: single and multi-stage (Dhavlikar et al. 2003; Khayet \& Cojocaru 2012). In a multi-stage approach, simulation runs are repeated by modifying the bounds of each independent variable considering the near optimum solution obtained in the previous simulation run, which is illustrated in Fig. 1.
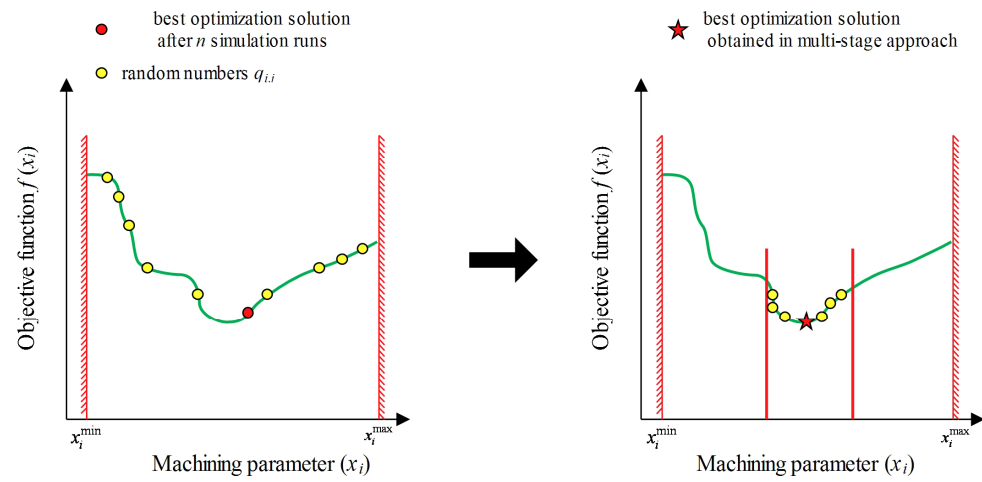

Fig. 1. Illustration of MC optimization procedure in single and multi-stage

The basic idea of the multi-stage MC method for solving optimization problems is given in Fig. 2.

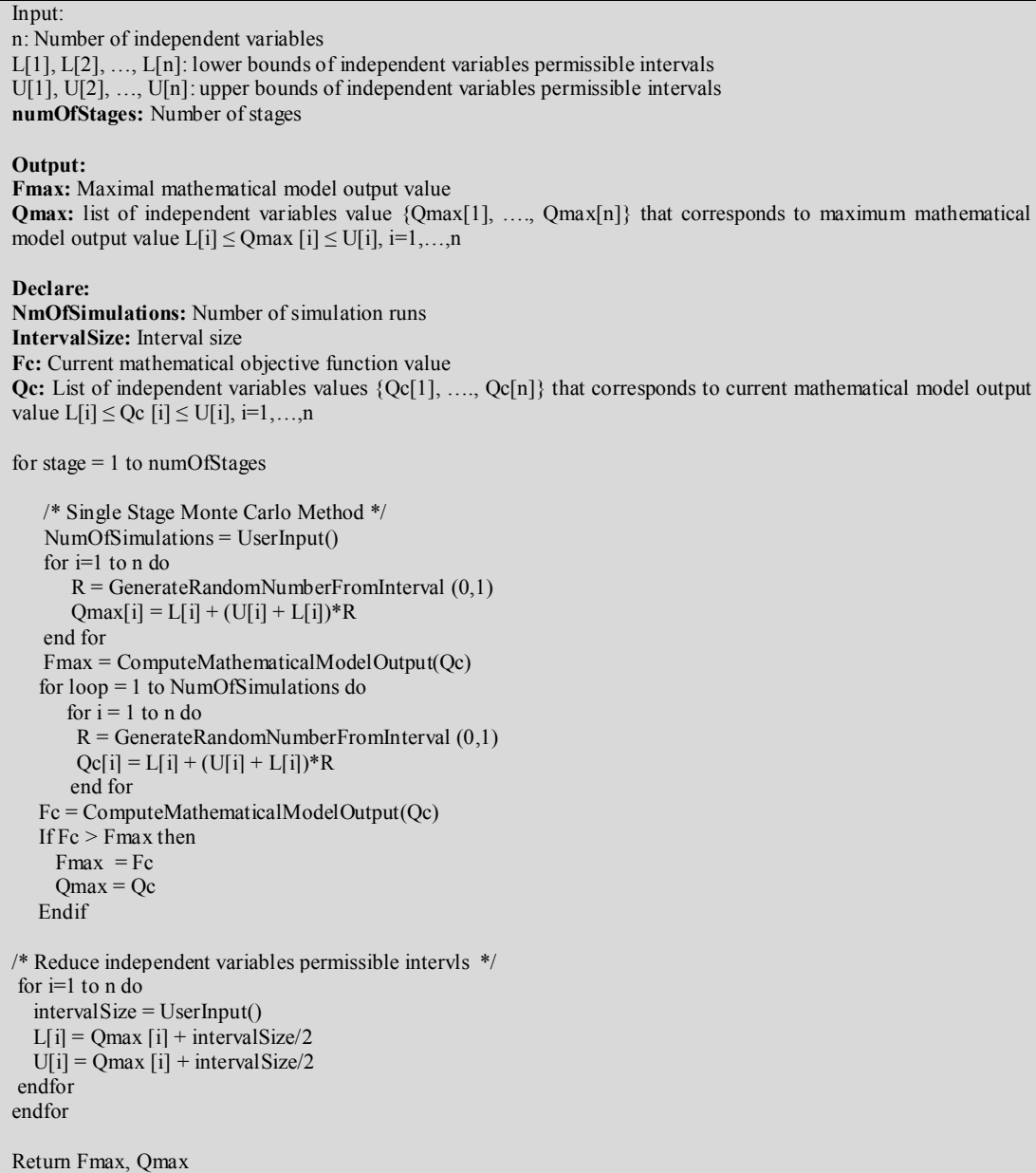

Fig. 2. Pseudo-code of the multi-stage MC method for solving optimization problems 


\section{Application examples}

The machining optimization application examples considered in this paper are taken from scientific resource bases, such as Springer and Elsevier. Case studies focused on solving machining optimization problems using meta-heuristic algorithms were chosen for comparative analysis of the obtained solutions by the multi-stage MC method. In order to facilitate the validation of obtained optimization solutions, the search for papers was restricted to only those dealing with mathematical models developed using regression analysis. In sum, six optimization case studies were considered - two case studies deal with three, two case studies deal with four and two case studies deal with five independent variables, i.e. machining parameters.

\subsection{Application of Monte Carlo method for solving machining optimization problems}

In solving machining optimization problems the aim of applying the MC method is to explore the optimization space and identify near optimal values of independent variables $\left(x_{i}\right)$ i.e. machining parameters such that objective function $f\left(x_{i}\right)$ has an extreme value (maximal or minimal) and the constraints for machining parameters $x_{i} \in\left[x_{i}^{\min }, x_{i}^{\max }\right]$ are satisfied. The machining optimization procedure based on the MC method was implemented in the MS Excel software package. The idea behind the MC method was to explore the space of feasible solutions by performing a large number of computational runs using pseudo-random numbers. After the formulation of a machining optimization problem, the first step in optimization procedure involves generation of random numbers $r_{i, j}$ uniformly distributed in the range $[0,1]$ using the function rand. Subsequently, in order to satisfy the bounds of machining parameters values, random numbers $r_{i, j}$ were used to generate random numbers $q_{i, j}$ for each machining parameter by using the following equation:

$$
q_{i, j}=x_{i}^{\min }+\left(x_{i}^{\max }-x_{i}^{\min }\right) \cdot r_{i, j}
$$

In such a way, by using random numbers $q_{i, j}$ and a mathematical model, a number of stochastic computational runs $(n)$ can be performed. When applying the single-stage approach, for each case study different numbers of simulation runs were performed, that is 1000, 2000, 5000 and 10000. Each was repeated 10 times and the best optimization solutions were recorded. In sum, for each case study $\mathrm{n}=$ 180000 simulation runs were performed. A simple procedure for computing was implemented in the Excel software package resulting in these computations being performed within few seconds.

In order to improve obtained optimization solutions, the multi-stage approach was implemented. After 180000 simulation runs performed in the first stage the near optimal optimization solution was identified. Subsequently, the bounds of each independent variable were diminished, 10000 simulation runs were repeated and the best optimization solution was identified. Although the multi-stage approach essentially refers to multiple modifications of the bounds of each independent variable, in this paper only one was performed.

\subsection{Single objective machining optimization}

\subsubsection{Case study 1}

Sarkar et al. (2006) investigated the influence of applied voltage, electrolyte concentration and interelectrode gap on material removal rate (MRR), radial overcut (ROC) and thickness of heat affected zone (HAZ) in an ECDM process. Based on the experimental data of Sarkar et al. (2006), Samanta and Chakraborty (2011) developed the following three equations using uncoded values of machining parameters: 


$$
\begin{aligned}
M R R= & 4.96423-0.20418 \cdot x_{1}+0.09862 \cdot x_{2}+0.00851 \cdot x_{3}+0.00249 \cdot x_{1}^{2} \\
& -0.00086 \cdot x_{2}^{2}+0.00039 \cdot x_{3}^{2}-0.00181 \cdot x_{1} \cdot x_{2}-0.00104 \cdot x_{1} \cdot x_{3}+0.00125 \cdot x_{2} \cdot x_{3} \\
R O C= & 3.15622-0.08019 \cdot x_{1}-0.07678 \cdot x_{2}-0.00356 \cdot x_{3}+0.00069 \cdot x_{1}^{2} \\
& +0.00048 \cdot x_{2}^{2}+0.00016 \cdot x_{3}^{2}+0.00072 \cdot x_{1} \cdot x_{2}-0.00026 \cdot x_{1} \cdot x_{3}+0.00041 \cdot x_{2} \cdot x_{3} \\
H A Z= & 0.940335-0.019541 \cdot x_{1}-0.028638 \cdot x_{2}-0.003122 \cdot x_{3}+0.000147 \cdot x_{1}^{2} \\
& +0.000242 \cdot x_{2}^{2}+0.000017 \cdot x_{3}^{2}+0.000251 \cdot x_{1} \cdot x_{2}-0.000017 \cdot x_{1} \cdot x_{3}+0.000106 \cdot x_{2} \cdot x_{3}
\end{aligned}
$$

where $x_{1}=$ applied voltage, $x_{2}=$ electrolyte concentration, $x_{3}=$ inter-electrode gap.

In an attempt to obtain optimal machining parameter values for minimization of ROC and HAZ and maximization of MRR, Samanta and Chakraborty (2011) and Rao and Kalyankar (2011) applied the

\begin{tabular}{|c|c|c|c|c|c|c|c|c|c|c|c|c|}
\hline \multirow{2}{*}{ Machining parameters and bounds } & \multicolumn{3}{|c|}{$\mathrm{ABC}$ algorithm } & \multicolumn{3}{|c|}{ TLBO algorithm } & \multicolumn{3}{|c|}{ Single stage $\mathrm{MC}$} & \multicolumn{3}{|c|}{ Multi-stage MC } \\
\hline & MRR & ROC & HAZ & MRR & ROC & HAZ & MRR & ROC & HAZ & MRR & $\mathrm{ROC}$ & HAZ \\
\hline & 70 & 50 & 50 & 70 & 50 & 50 & 69.973 & 50.159 & 50.081 & 69.999 & 50.007 & 50.004 \\
\hline Elect & 20 & 30 & 24.5 & 10 & 30 & 25 & 10.299 & & & 10.002 & & 91 \\
\hline Inter-electrode $\mathrm{g}$ & 20 & 20 & 40 & 21 & 20 & 38 & 20.598 & & 38.4 & & & 39.994 \\
\hline Optimal value & $1.3372^{*}$ & 0.05912 & 0.05409 & 1.5902 & 0.0591 & 0.0541 & 1.5958 & 0.0611 & 0.0542 & 1.6252 & 0.05919 & 0.054092 \\
\hline
\end{tabular}
ABC and TLBO algorithm, respectively. The bounds of machining parameters used in optimization procedure as well as optimization solutions are given in Table 1. Optimization solutions obtained using MC methods are also shown in Table 1 as follows.

\section{Table 1}

Optimization solutions of case study 4.2.1

${ }^{*}$ Corrected value after Venkata Rao and Kalyankar (2011)

\subsubsection{Case study 2}

Kilickap et al. (2011) presented a study focused on the influence of machining parameters on the surface roughness obtained in drilling of AISI 1045. The mathematical relationship for correlating the surface roughness and the considered drilling parameters was obtained in the following form:

$$
R_{a}=4.115-0.82767 \cdot x_{1}+8.225 \cdot x_{2}+0.135 \cdot x_{3}+0.0538 \cdot x_{1}^{2}
$$

where $x_{1}=$ cutting speed, $x_{2}=$ feed rate, $x_{3}=$ cutting environment. In order to determine optimum drilling parameters for AISI 1045 material at the lowest possible surface roughness value, Kilickap et al. (2011) used GA. The bounds of machining parameters used and comparison of optimization solutions found by GA, PS algorithm (Madić \& Radovanović, 2014b) and the MC method are given in Table 2.

\section{Table 2}

Optimization solutions of case study 4.2.2

\begin{tabular}{lcccc}
\hline Machining parameters and bounds & GA & PS & Single stage MC & Multi-stage MC \\
\hline Cutting speed (m/min) [5 - 15] & 7.62 & 7.692 & 7.813 & 7.690 \\
Feed rate (mm/rev) $[0.1-0.3]$ & 0.1 & 0.1 & 0.1 & 0.1 \\
Cutting environment $[1-3]$ & 1 & 1 & 1.016 & 1 \\
Ra optimal value & 1.89 & 1.8892 & 1.8958 & 1.8892 \\
\hline
\end{tabular}

\subsubsection{Case study 3}

Bhushan et al. (2012) investigated turning of Al Alloy SiC particle composite material using carbide turning inserts. The investigation was focused on the application of GA for finding the optimal machining parameters at which surface roughness has minimal value. Bhushan et al. (2012) developed the following mathematical model relating the machining parameters and surface roughness: 


$$
\begin{aligned}
R_{a}= & 0.72412+0.00324 \cdot x_{1}-0.19694 \cdot x_{2}+4.19915 \cdot x_{3}-0.18753 \cdot x_{4}-0.0000174 \cdot x_{1}^{2} \\
& -3.42419 \cdot x_{3}^{2}+3.33125 \cdot x_{2} \cdot x_{3}-0.56484 \cdot x_{3} \cdot x_{4}
\end{aligned}
$$

where $x_{1}=$ cutting speed, $x_{2}=$ feed rate, $x_{3}=$ depth of cut and $x_{4}=$ tool nose radius. The bounds of machining parameters used in optimization procedure and comparison of optimization solutions found by Bhushan et al. (2012) by using GA to those obtained by using the MC method are given in Table 3. The same optimization problem was solved previously by Madić and Radovanović (2014b) by using the PS algorithm.

Table 3

Optimization solutions of case study 4.2.3

\begin{tabular}{lcccc}
\hline Parameters and objective function & GA & PS & Single stage MC & Multi-stage MC \\
\hline Cutting speed (m/min) [90-210] & 207.055 & 210 & 206.198 & 209.867 \\
Feed rate $(\mathrm{mm} / \mathrm{rev})[0.15-0.25]$ & 0.151 & 0.15 & 0.152 & 0.152 \\
Depth of cut (mm) [0.2-0.6] & 0.201 & 0.2 & 0.208 & 0.2 \\
Tool nose radius $(\mathrm{mm})[0.4-1.2]$ & 1.199 & 1.2 & 1.197 & 1.2 \\
\hline Surface roughness & $1.06509^{*}$ & 1.0498 & 1.0885 & 1.0516 \\
\hline *
\end{tabular}

Corrected values

\subsubsection{Case study 4}

Savas and Ozay (2008) presented the GA approach for optimization of cutting parameters at cylindrical workpieces leading to minimal surface roughness in the tangential turn-milling process. The mathematical model for the prediction of surface roughness considering machining parameters was obtained according to experiment results and is given in the equation below:

$$
\begin{aligned}
R_{a}= & \left(0.000008 \cdot x_{1}^{2}-0.0082 \cdot x_{1}+2.8734\right) \cdot\left(0.00003 \cdot x_{2}^{2}-0.0135 \cdot x_{2}+1.9924\right) . \\
& \cdot\left(0.0171 \cdot x_{3}+0.4677\right) \cdot\left(0.2525 \cdot x_{4}+0.4087\right) \cdot 5.3
\end{aligned}
$$

where $x_{1}=$ workpiece speed, $x_{2}=$ tool speed, $x_{3}=$ feed rate and $x_{4}=$ depth of cut.

The optimization solution for turn-milling process obtained by Savas and Ozay (2008) by using GA is given in Table 4. In the same table the bounds of machining parameters used in optimization procedure and optimization solution obtained by the MC method are given. The same optimization problem was solved previously by Madić and Radovanović (2014b) by using the PS algorithm.

\section{Table 4}

Optimization solutions of case study 4.2.4

\begin{tabular}{lcccc}
\hline Parameters and objective function & GA & PS & Single stage MC & Multi-stage MC \\
Workpiece speed (rev/min) [300 - 700] & 511.9 & 512.5 & 497.716 & 513.305 \\
Tool speed (rev/min) [150-300] & 224.9 & 225 & 225.164 & 226.193 \\
Feed rate (mm/min) [3 - 20] & 3.2 & 3 & 3.055 & 3 \\
Depth of cut (mm) [0.1 - 1] & 0.1 & 0.1 & 0.106 & 0.1 \\
Surface roughness & $0.4394^{*}$ & 0.4366 & 0.4398 & 0.4366 \\
\hline
\end{tabular}

${ }^{*}$ Corrected values

\subsubsection{Case study 5}

Çaydaş and Hasçalik (2008) investigated the relationship between machining parameters and surface roughness in AWJ machining of Al 7075-T6 wrought alloy. Based on experimental data, the following prediction model was developed:

$$
\begin{aligned}
R_{a}= & -5.07976+0.08169 \cdot V+0.07912 \cdot P-0.34221 \cdot h-0.08661 \cdot d \\
& -0.34866 \cdot m-0.00031 \cdot V^{2}-0.00012 \cdot P^{2}+0.10575 \cdot h^{2} \\
& +0.00041 \cdot d^{2}+0.07590 \cdot m^{2}-0.00008 \cdot V \cdot m-0.00009 \cdot P \cdot m \\
& +0.03089 \cdot h \cdot m+0.00513 \cdot d \cdot m
\end{aligned}
$$


where $x_{1}=$ traverse speed, $x_{2}=$ waterjet pressure, $x_{3}=$ standoff distance, $x_{4}=$ abrasive grit size and $x_{5}=$ abrasive flow rate. Zain et al. (2011) used Eq. (7) as the objective function to determine optimal values of machining parameters so as to minimize surface roughness by using GA and SA. Recently, for solving the same problem, Yusup et al. (2012) and Madić and Radovanović (2014b) attempted the $\mathrm{ABC}$ and PS algorithm, respectively. The bounds of machining parameters used in optimization procedure and optimization solutions obtained by past researchers and those obtained by using the MC method are given in Table 5.

\section{Table 5}

Comparison of optimization solutions of case study 4.2.5

\begin{tabular}{|c|c|c|c|c|c|c|}
\hline Optimization approach & $\begin{array}{c}\text { Traverse speed } \\
(\mathrm{mm} / \mathrm{min}) \\
{[50-150]}\end{array}$ & $\begin{array}{c}\text { Waterjet pressure } \\
(\mathrm{MPa}) \\
{[125-250]}\end{array}$ & $\begin{array}{c}\text { Standoff } \\
\text { distance }(\mathrm{mm}) \\
{[1-4]}\end{array}$ & $\begin{array}{c}\text { Abrasive grit size } \\
(\mu \mathrm{m}) \\
{[60-120]}\end{array}$ & $\begin{array}{c}\text { Abrasive flow } \\
\text { rate }(\mathrm{g} / \mathrm{s}) \\
{[0.5-3.5]}\end{array}$ & $\begin{array}{c}\text { Minimal } \\
R_{a}\end{array}$ \\
\hline$\overline{\mathrm{GA}}$ & 50.024 & 125.018 & 1.636 & 94.73 & 0.525 & 1.554 \\
\hline SA & 50.003 & 125.029 & 1.486 & 107.737 & 0.5 & 1.533 \\
\hline $\mathrm{ABC}$ & 50 & 125 & 1.550 & 102.521 & 0.5 & 1.522 \\
\hline PS & 50 & 125 & 1.545 & 102.494 & 0.5 & 1.5223 \\
\hline Single stage $\mathrm{MC}$ & 50.610 & 126.443 & 1.125 & 103.096 & 0.506 & 1.64392 \\
\hline Multi-stage MC & 50.030 & 125.005 & 1.255 & 101.778 & 0.501 & 1.53351 \\
\hline
\end{tabular}

\subsubsection{Case study 6}

Munda and Bhattacharyya (2008) presented an approach for investigation of the EMM process using RSM. Five machining parameters, pulse on/off ratio, machining voltage, electrolyte concentration, voltage frequency and tool vibration frequency and two machining performance characteristics such as MRR and ROC were considered in the study. The RSM mathematical models for MRR and ROC prediction on the basis of machining parameters are given in Eqs. (8a) and (8b), respectively:

$$
\begin{aligned}
& M R R=-1.78917+0.111858 \cdot x_{1}+1.36263 \cdot x_{2}-0.0864044 \cdot x_{3}+0.0231122 \cdot x_{4} \\
& -0.00139639 \cdot x_{5}-0.201666 \cdot x_{1}^{2}-0.0860582 \cdot x_{2}^{2}-0.000145752 \cdot x_{3}^{2}-0.000319532 \cdot x_{4}^{2} \\
& +3.893684 \cdot 10^{-6} \cdot x_{5}^{2}-0.0704326 \cdot x_{1} \cdot x_{2}+0.00838936 \cdot x_{1} \cdot x_{3}+0.00275664 \cdot x_{1} \cdot x_{4} \\
& +0.00178484 \cdot x_{1} \cdot x_{5}+0.00870264 \cdot x_{2} \cdot x_{3}-0.00700764 \cdot x_{2} \cdot x_{4}-0.00105004 \cdot x_{2} \cdot x_{5} \\
& +0.00125437 \cdot x_{3} \cdot x_{4}+0.0000247626 \cdot x_{3} \cdot x_{5}+0.0000181174 \cdot x_{4} \cdot x_{5} \\
& R O C=-1.08149+1.21039 \cdot x_{1}+0.448639 \cdot x_{2}-0.0821333 \cdot x_{3}+0.0247783 \cdot x_{4} \\
& -0.00258589 \cdot x_{5}+0.0198541 \cdot x_{1}^{2}+0.0554876 \cdot x_{2}^{2}+0.00108447 \cdot x_{3}^{2}+0.000640329 \cdot x_{4}^{2} \\
& +2.817205 \cdot 10^{-6} \cdot x_{5}^{2}-0.139966 \cdot x_{1} \cdot x_{2}-0.00133867 \cdot x_{1} \cdot x_{3}-0.0161759 \cdot x_{1} \cdot x_{4} \\
& -5.41335 \cdot 10^{-5} \cdot x_{1} \cdot x_{5}-0.00307591 \cdot x_{2} \cdot x_{3}-0.0163201 \cdot x_{2} \cdot x_{4}+0.000831331 \cdot x_{2} \cdot x_{5} \\
& +0.000786541 \cdot x_{3} \cdot x_{4}+0.0000725981 \cdot x_{3} \cdot x_{5}-6.94181 \cdot 10^{-5} \cdot x_{4} \cdot x_{5}
\end{aligned}
$$

where $x_{1}=$ pulse on/off ratio, $x_{2}=$ machining voltage, $x_{3}=$ electrolyte concentration, $x_{4}=$ voltage frequency and $x_{5}=$ tool vibration frequency. In order to investigate the applicability and suitability of the ABC algorithm for solving machining optimization problems, Samanta and Chakraborty (2011) used Eqs. (8a) and (8b) as the objective functions to determine optimal values of machining parameters. The bounds of machining parameters used in optimization procedure and optimization results of single objective optimization of the MRR and ROC for the EMM process obtained by the ABC algorithm and MC method are given in Table 6.

As seen from Table 6, optimization of mathematical model for ROC (Eq. 8b) using MC method yielded a negative value as the optimal one. It is recognized that the negative value of ROC is physically meaningless, however, this value is regarded only as the optimal solution of the mathematical model. Although it was observed by Munda and Bhattacharyya (2008) that the mathematical model for ROC 
prediction is statistically adequate at $97.5 \%$ confidence level, it is observed that there is no guarantee that the model will yield meaningful predictions over the entire experimentally investigated range.

Table 6

Optimization solutions of case study 4.2.6

\begin{tabular}{lcccccc}
\hline \multirow{2}{*}{ Machining parameters and bounds } & \multicolumn{2}{c}{ ABC algorithm } & \multicolumn{2}{c}{ Single stage MC } & \multicolumn{2}{c}{ Multi-stage MC } \\
& MRR & ROC & MRR & ROC & MRR & ROC \\
\hline Pulse on/off ratio [0.5-2.5] & 0.9168 & 0.6288 & 0.912 & 0.511 & 0.966 & 0.501 \\
Machining voltage (V) [2.5-4.5] & 4.5 & 3.2639 & 4.499 & 2.534 & 4.498 & 2.501 \\
Electrolyte concentration (g/l) [10-30] & 30 & 18.7930 & 29.872 & 20.766 & 29.996 & 20.546 \\
Voltage frequency (Hz) [35-55] & 52.4956 & 39.4123 & 54.923 & 35.663 & 54.606 & 35.012 \\
Tool vibration frequency (Hz) [100-300] & 100 & 249.4320 & 108.436 & 270.838 & 105.011 & 270.287 \\
Optimal value & 1.47 & 0 & 1.4483 & -0.2424 & 1.4584 & -0.2649 \\
\hline
\end{tabular}

\subsection{Analysis and discussion}

As it can be seen from the results in the previous section, the identified near optimal machining conditions are about the same as those selected as optimal by previous researches. Given that the optimization was based on using mathematical models as objective functions, all optimization solutions obtained by the multi-stage MC method can be verified through a trivial calculation using mathematical models given in section 4. Considering stochastic nature of the MC method and meta-heuristic methods, validation and possible improvement of optimization solutions can be performed using exhaustive search algorithm. In our previous work (Kovačević et al. 2013) the possibilities and advantages of applying exhaustive iterative search for solving machining optimization problems and validation of optimization results obtained by other optimization methods were presented.

A deeper analysis of the machining optimization solutions indicates that in the case of mathematical models with up to four independent variables, the optimization solutions obtained by the multi-stage MC method are comparable or even better than solutions obtained by past researchers using metaheuristic algorithms such as GA, ABC and TLBO. For the case study 5, which deals with five independent variables, it was observed that the optimization solution obtained using the multi-stage MC method is better that the optimization solutions obtained by GA and SA, but poorer than the one obtained by $\mathrm{ABC}$ algorithm. A comparative analysis of optimization solutions of case study 4.2.6 in the case of five independent variables reveals that the multi-stage MC method is less effective in high dimensional spaces, and the advantage should be given to the $\mathrm{ABC}$ algorithm.

When applying the MC method for solving machining optimization problems it should be noted that a decrease in the variability of obtained solutions was not observed with an increase in the number of simulation runs. Also, it was observed that in some cases e.g. (case study 1) the best optimization solution was obtained after only 5000 simulation runs, whereas in some cases the best optimization solution was obtained after a larger number of simulation runs. In other words, there is no greater certainty that the better optimization solution will be found with an increasing number of simulation runs, which is the case when using meta-heuristics. The impossibility to decrease the variability of obtained solutions and increase certainty of obtaining better optimization solutions with increasing number of simulation runs is a consequence of the fact that the MC method does not use any search guide information and rules, but only random numbers. However, possible improvements of optimization solutions might be expected by a drastic increase in the number of simulation runs.

The optimization solutions presented in this paper indicate that the multi-stage MC method involving few thousand $\mathrm{MC}$ computation runs represents a fairly effective and efficient approach for solving multi-dimensional machining optimization problems. Furthermore, the MC based optimization approach is very simple to implement, requires no expert knowledge of optimization theory and artificial intelligence, setting of algorithm parameters and/or defining an initial solution as in the case of using other optimization methods. 
The salient advantage of the application of the MC method is that it is possible to obtain a population of optimization solutions. Graphical visualization of the optimization solutions for the case study 4.2.1. (maximization of MRR) obtained using the multi-stage MC method are shown in Figure 3. By plotting these graphs, all acceptable solutions with corresponding values of machining parameters can be easily identified. This is particularly advantageous in machining practice considering different machine/tool constraints and limitations.

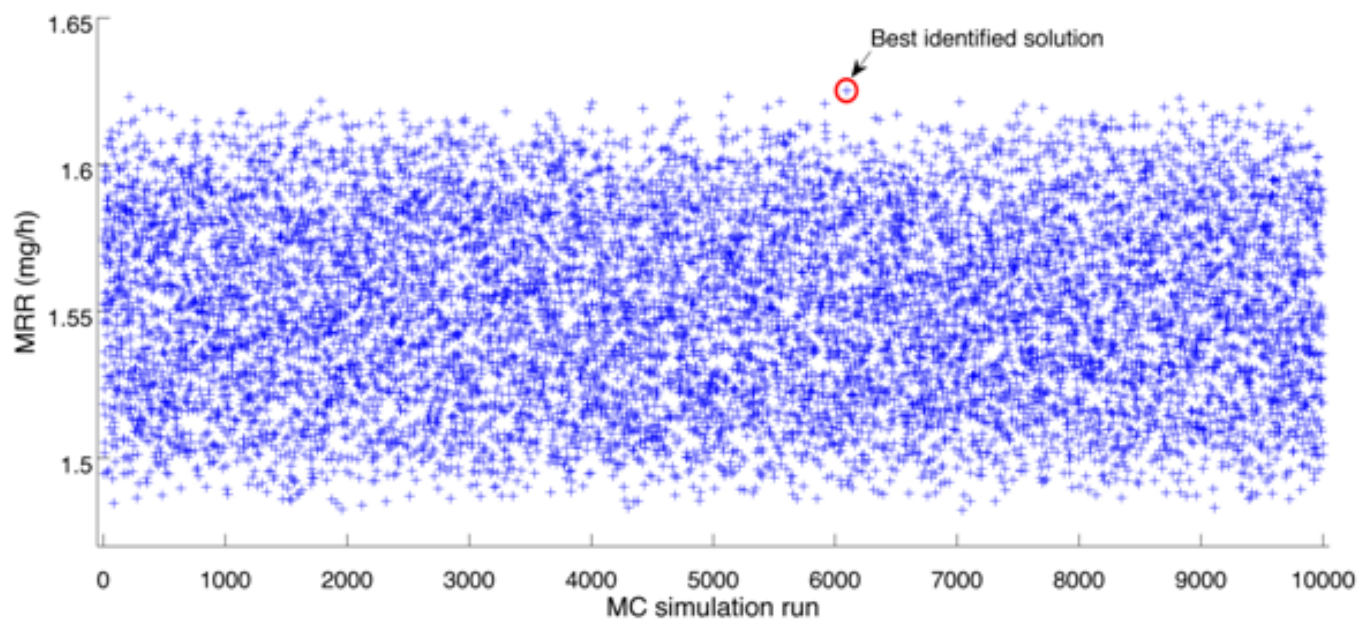

Fig. 3. Optimization solutions obtained using the multi-stage MC method for the case study 4.2.1.

\section{Conclusion}

This paper presented an investigation of applicability of the multi-stage MC method for solving machining optimization problems. To this aim six machining optimization problems taken from relevant literature were considered and the obtained results were compared with the results obtained by previous researchers using different meta-heuristic optimization methods. The optimization procedure based on the MC method is very simple, both conceptually and in implementation without the need to write a programming code or use specialized software packages, and by the authors' opinion represents an optimization tool being most suited to use by process engineers in real production environment. The salient features of MC include the following: (i) solving optimization problems requires no expert knowledge of optimization theory, artificial intelligence, setting of different algorithm parameters and/or defining an initial solution as in the case of using other optimization methods, (ii) there is a possibility to find a population of optimization solutions and rank them, (iii) the multi-stage approach provides the possibility for an improvement of the optimization solutions. The analysis of the obtained results can be summarized by the following points:

- in the case of mathematical models with up to four independent variables, the optimization solutions obtained by the multi-stage MC method are comparable with the optimization solutions obtained by using other meta-heuristic optimization methods,

- the multi-stage MC method may not be suitable for higher dimensional spaces and the priority for solving machining optimization problems should be given to meta-heuristic optimization methods which have powerful mathematical tools that guide the exploration of optimization space. However, the optimization solutions obtained using the MC method can be used as initial solutions such as in the case of SA,

- the MC method proves its applicability as a very simple optimization method for solving machining optimization problems. 
The main scope of our future work will be the application of the multi-stage MC method for solving multi-objective machining optimization problems. Furthermore, attempts will be made to investigate the applicability of a combined optimization approach by integrating the MC method with the brute force algorithm based on an exhaustive iterative search. It is believed that the application of this hybrid approach for solving machining optimization problems in continual search space will outperform the optimization results of other meta-heuristic optimization methods in terms of solution accuracy and computational speed.

\section{Acknowledgements}

This work was carried out within the project TR 35034 financially supported by the Ministry of Education and Science of the Republic of Serbia.

\section{References}

Besseris, G. J. (2008). Multi-response optimisation using Taguchi method and super ranking concept. Journal of Manufacturing Technology Management,19(8), 1015-1029.

Besseris, G. J. (2008). Multi-response optimisation using Taguchi method and super ranking concept. Journal of Manufacturing Technology Management,19(8), 1015-1029.

Çaydaş, U., \& Hasçalık, A. (2008). A study on surface roughness in abrasive waterjet machining process using artificial neural networks and regression analysis method. Journal of materials processing technology, 202(1), 574-582.

Davim, J. P. (2001). A note on the determination of optimal cutting conditions for surface finish obtained in turning using design of experiments. Journal of materials processing technology, 116(2), 305-308.

Dhavlikar, M. N., Kulkarni, M. S., \& Mariappan, V. (2003). Combined Taguchi and dual response method for optimization of a centerless grinding operation.Journal of Materials Processing Technology, 132(1), 90-94.

Khayet, M., \& Cojocaru, C. (2012). Artificial neural network modeling and optimization of desalination by air gap membrane distillation. Separation and Purification Technology, 86, 171-182.

Kilickap, E., Huseyinoglu, M., \& Yardimeden, A. (2011). Optimization of drilling parameters on surface roughness in drilling of AISI 1045 using response surface methodology and genetic algorithm. The International Journal of Advanced Manufacturing Technology, 52(1-4), 79-88.

Kovačević, M., Madić, M., \& Radovanović, M. (2013). Software prototype for validation of machining optimization solutions obtained with meta-heuristic algorithms. Expert Systems with Applications, 40(17), 6985-6996.

Kroese, D. P., Taimre, T., \& Botev, Z. I. (2011). Handbook of Monte Carlo Methods (Vol. 706). John Wiley \& Sons.

Madić, M., Marković, D., \& Radovanović, M. (2013). Comparison of meta-heuristic algorithms for solving machining optimization problems. Facta universitatis-series: Mechanical Engineering, 11(1), 29-44.

Madić, M., \& Radovanović, M. (2014a). Possibilities of using Monte Carlo method for solving machining optimization problems. Facta Universitatis, Series: Mechanical Engineering, 12(1), 2736.

Madić M, Radovanović M (2014b) Optimization of machining processes using pattern search algorithm. International Journal of Industrial Engineering Computations, 5(2), 223-234.

Mosegaard, K., \& Sambridge, M. (2002). Monte Carlo analysis of inverse problems. Inverse Problems, 18(3), 29-34.

Mukherjee, I., \& Ray, P. K. (2006). A review of optimization techniques in metal cutting processes. Computers \& Industrial Engineering, 50(1), 15-34. 
Munda, J., \& Bhattacharyya, B. (2008). Investigation into electrochemical micromachining (EMM) through response surface methodology based approach. The International Journal of Advanced Manufacturing Technology,35(7-8), 821-832.

Rao, R. V., Pawar, P. J., \& Shankar, R. (2008). Multi-objective optimization of electrochemical machining process parameters using a particle swarm optimization algorithm. Proceedings of the Institution of Mechanical Engineers, Part B: Journal of Engineering Manufacture, 222(8), 949-958.

Rao, R. V., \& Pawar, P. J. (2009). Modelling and optimization of process parameters of wire electrical discharge machining. Proceedings of the Institution of Mechanical Engineers, Part B: Journal of Engineering Manufacture, 223(11), 1431-1440.

Venkata Rao, R., \& Pawar, P. J. (2010). Parameter optimization of a multi-pass milling process using non-traditional optimization algorithms. Applied soft computing, 10(2), 445-456.

Rao, R. V., \& Kalyankar, V. D. (2011). Parameters optimization of advanced machining processes using TLBO algorithm. EPPM, Singapore, 20, 21-31

Venkata Rao, R., \& Kalyankar, V. D. (2013). Parameter optimization of modern machining processes using teaching-learning-based optimization algorithm. Engineering Applications of Artificial Intelligence, 26(1), 524-531.

Samanta, S., \& Chakraborty, S. (2011). Parametric optimization of some non-traditional machining processes using artificial bee colony algorithm. Engineering Applications of Artificial Intelligence, 24(6), 946-957.

Sarkar, B. R., Doloi, B., \& Bhattacharyya, B. (2006). Parametric analysis on electrochemical discharge machining of silicon nitride ceramics. The International Journal of Advanced Manufacturing Technology, 28(9-10), 873-881.

Savas, V., \& Ozay, C. (2008). The optimization of the surface roughness in the process of tangential turn-milling using genetic algorithm. The International Journal of Advanced Manufacturing Technology, 37(3-4), 335-340.

Yang, X. S. (2010). Engineering optimization: an introduction with metaheuristic applications. John Wiley \& Sons.

Y1ldiz, A. R. (2009). A novel particle swarm optimization approach for product design and manufacturing. The International Journal of Advanced Manufacturing Technology, 40(5-6), 617628.

Yusup, N., Zain, A. M., \& Hashim, S. Z. M. (2012). Evolutionary techniques in optimizing machining parameters: Review and recent applications (2007-2011).Expert Systems with Applications, 39(10), 9909-9927.

Yusup, N., Sarkheyli, A., Zain, A. M., Hashim, S. Z. M., \& Ithnin, N. (2013). Estimation of optimal machining control parameters using artificial bee colony.Journal of Intelligent Manufacturing, 1-10.

Zain, A. M., Haron, H., \& Sharif, S. (2011). Genetic algorithm and simulated annealing to estimate optimal process parameters of the abrasive waterjet machining. Engineering with computers, 27(3), 251-259. 